\title{
OBLIQUE WATER WAVE DIFFRACTION BY A STEP
}

\author{
P. DOLAI \\ Department of Mathematics \\ Prasannadeb Women's College \\ Jalpaiguri-735101, West Bengal, INDIA \\ E-mail: dolaiprity68@gmail.com
}

\begin{abstract}
This paper is concerned with the problem of diffraction of an obliquely incident surface water wave train on an obstacle in the form of a finite step. Havelock expansions of water wave potentials are used in the mathematical analysis to obtain the physical parameters reflection and transmission coefficients in terms of integrals. Appropriate multi-term Galerkin approximations involving ultraspherical Gegenbauer polynomials are utilized to obtain a very accurate numerical estimate for reflection and transmission coefficients which are depicted graphically. From these figures various interesting results are discussed.
\end{abstract}

Key words: finite step, Havelock expansion, Galerkin approximation, Gegenbauer polynomial, reflection and transmission coefficients.

\section{Introduction}

Mathematical study of water wave diffraction problems involving fixed vertical thin barriers of various configurations was initiated long back using a variety of mathematical methods (cf. Dean [1], Ursell [2], Evans [3], Porter [4], Mandal and Dolai [5], etc.). The problems of water wave scattering by an irregular bottom have received some considerable interest in the literature on linearised theory of water waves due to their importance in finding the effects of naturally occurring bottom obstacles such as sand ripples on the wave motion (cf. Roseau [6], Kreisel [7], Fitz Gerald [8], Hamilton [9], Newman [10], Miles [11], Mandal and Gayen [12], Dolai and Dolai [13]).

Problems involving the propagation of water waves in a fluid of variable depth can be divided into three categories: 'beach' problems, where the depth tends to zero, 'obstacle' problems, where the depth is a constant except for variations extending over a finite interval in space, and 'changing-depth' problems, where the depth changes from one limiting value to a second limiting value. There have been many investigations of the beach and obstacle problems (cf. Stoker [14], Wehausen and Laitone [15]), but comparatively few studies have been made on the 'changing depth' case (cf. Bartholomeusz [16], Evans and McIver [17], Newman [10]). The importance of wave propagation in the case of changing depth is obvious in many coastal situations such as the passage of waves over a continental shelf. As an idealization of such a problem, we consider here the case of wave propagation over a finite step, from one constant finite depth to another.

In the present paper we consider the problem of diffraction of an obliquely incident surface wave train on an obstacle in the form of a finite step, from one constant finite depth to another. By a suitable application of Havelock's expansions of water wave potentials, we obtain the reflection and transmission coefficients in terms of integrals. Appropriate multi-term Galerkin approximations involving ultraspherical Gegenbauer polynomials are utilized to obtain a very accurate numerical estimate for reflection and transmission coefficients which are depicted graphically. From these figures various interesting results are discussed. 


\section{Formulation of the problem}

We consider the motion in an inviscid, homogeneous, incompressible liquid which is supposed to be confined between two vertical planes perpendicular to the edges of the step. Cartesian axes are chosen with the mean free surface, the $(x, z)$ plane with the origin directly above the step, while the axis of $y$ is directed down wards into the liquid. The shallower water is of depth $h_{l}$ above the horizontal shelf, the deeper water is of depth $h_{2}$. A simple sketch of the problem is given in Fig.1.

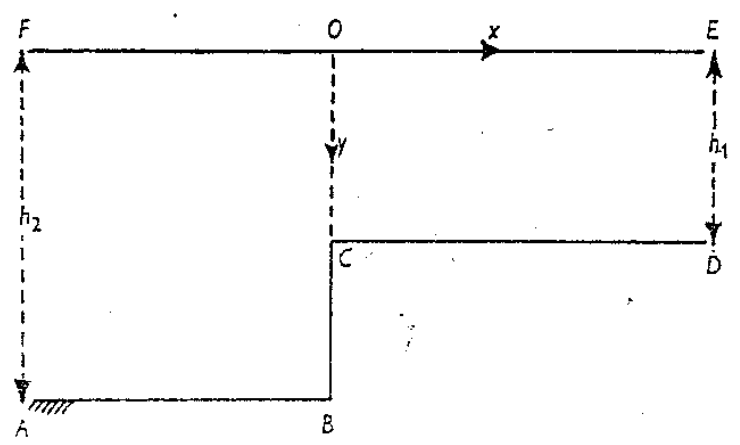

Fig.1. Geometry of the problem.

A simple harmonic progressive oblique wave train originating at $x \rightarrow-\infty$ is incident on the step, and is partially reflected and partially transmitted. Assuming linear theory, the time harmonic progressive waves from negative infinity can be represented by the velocity potentials $\operatorname{Re}\left\{\varphi_{-}^{\text {inc }}(x, y) \exp (i \vartheta z-i \sigma t)\right\}$ where

$$
\varphi_{-}^{\text {inc }}(x, y)=\frac{\cosh k_{0}\left(h_{2}-y\right)}{\cosh k_{0} h_{2}} \exp (i \mu x)
$$

$k_{0}$ being the real positive root of

$$
k \tanh k h_{2}=K,
$$

with $K=\sigma^{2} / g, \vartheta=k_{0} \sin \alpha, \mu=k_{0} \cos \alpha, \sigma$ being the frequency of the incoming waves and $g$ being the gravity. Due to the presence of the step, the oblique incident wave train is partially reflected by the step and partially transmitted through the gap. If the resulting motion is described by the velocity potential $\mathrm{Re}$ $\{\varphi(x, y) \exp (i \vartheta z-i \sigma t)\}$, then $\varphi$ satisfies

$$
\nabla^{2} \varphi-\vartheta^{2} \varphi=0 \quad \text { in the fluid region }
$$

the free surface condition

$$
K \varphi+\frac{\partial \varphi}{\partial y}=0 \quad \text { on } \quad y=0
$$

the bottom condition 


$$
\frac{\partial \varphi}{\partial y}=0 \quad \text { on } \quad y=h_{1}, \quad x>0 \quad \text { and } \quad y=h_{2}, \quad x<0
$$

the condition on the step

$$
\frac{\partial \varphi}{\partial x}=0 \quad \text { on } \quad x=0-, \quad y \in L\left(h_{1}<y \leq h_{2}\right)
$$

the edge condition

$$
r^{1 / 3} \nabla \varphi \quad \text { is bounded as } \quad r \rightarrow 0
$$

$r$ is the distance from the edge, and the infinity condition

$$
\varphi(x, y) \rightarrow\left\{\begin{array}{l}
T_{1} \frac{\cosh \alpha_{0}\left(h_{1}-y\right)}{\cosh \alpha_{0} h_{1}} \exp (i \beta x) \text { as } x \rightarrow \infty \\
\frac{\cosh k_{0}\left(h_{2}-y\right)}{\cosh k_{0} h_{2}}\left\{\exp (i \mu x)+R_{1} \exp (-i \mu x)\right\} \text { as } x \rightarrow-\infty
\end{array}\right.
$$

where $\alpha_{0}$ satisfies $\alpha_{0} \tanh \alpha_{0} h_{1}=K, \quad \beta^{2}=\alpha_{0}^{2}-\vartheta^{2}$ and $T_{1}, R_{1}$ are the unknown transmission and reflection coefficients to be determined.

\section{Method of solution}

Since $\varphi_{x}(x, y)$ and $\varphi(x, y)$ are continuous across OC, we can write

$$
\begin{aligned}
& \left(\frac{\partial \varphi}{\partial x}\right)_{x=0+}=\left(\frac{\partial \varphi}{\partial x}\right)_{x=0-}=f(y), \text { say, for } 0<y<h_{l}, \\
& (\varphi)_{x=0+}=(\varphi)_{x=0-} \text { for } 0<y<h_{1} .
\end{aligned}
$$

A solution for $\varphi(x, y)$ satisfying Eqs (2.3), (2.4), (2.5) and (2.8) can be represented as

$$
\varphi(x, y) \rightarrow \begin{cases}T_{1} \frac{\cosh \alpha_{0}\left(h_{1}-y\right)}{\cosh \alpha_{0} h_{1}} \exp (i \beta x)+\sum_{1}^{\infty} A_{n} \cos \alpha_{n}\left(h_{1}-y\right) \exp \left(-s_{n} x\right), \quad x \geq 0, \\ \frac{\cosh k_{0}\left(h_{2}-y\right)}{\cosh k_{0} h_{2}}\left\{\exp (i \mu x)+R_{1} \exp (-i \mu x)\right\}+\sum_{1}^{\infty} B_{n} \cos k_{n}\left(h_{2}-y\right) \exp \left(p_{n} x\right), \quad x \leq 0\end{cases}
$$

where $s_{n}^{2}=\alpha_{n}^{2}+\vartheta^{2}, p_{n}^{2}=k_{n}^{2}+\vartheta^{2}, \alpha_{n}$ satisfy $\alpha_{n} \tan \alpha_{n} h_{1}+K=0, k_{n}$ satisfy $k_{n} \tan k_{n} h_{2}+K=0$.

Using Eq.(3.3) in Eqs (3.1) and (3.2), we find 


$$
\begin{aligned}
& f(y)=i \beta T_{1} \frac{\cosh \alpha_{0}\left(h_{1}-y\right)}{\cosh \alpha_{0} h_{1}}-\sum_{1}^{\infty} s_{n} A_{n} \cos \alpha_{n}\left(h_{1}-y\right), \quad 0<y<h_{1}, \\
& =i \mu\left(1-R_{1}\right) \frac{\cosh k_{0}\left(h_{2}-y\right)}{\cosh k_{0} h_{2}}+\sum_{l}^{\infty} p_{n} B_{n} \cos k_{n}\left(h_{2}-y\right), \quad 0<y<h_{2},
\end{aligned}
$$

and

$$
\begin{aligned}
& T_{1} \frac{\cosh \alpha_{0}\left(h_{1}-y\right)}{\cosh \alpha_{0} h_{1}}+\sum_{l}^{\infty} A_{n} \cos \alpha_{n}\left(h_{1}-y\right)=\left(1+R_{1}\right) \frac{\cosh k_{0}\left(h_{2}-y\right)}{\cosh k_{0} h_{2}}+ \\
& +\sum_{l}^{\infty} B_{n} \cos k_{n}\left(h_{2}-y\right), \quad 0<y<h_{1} .
\end{aligned}
$$

The use of Havelock's [18] inversion theorem in Eqs (3.4) produces

$$
\begin{aligned}
& i \beta T_{1}=\frac{4 \alpha_{0} \cosh \alpha_{0} h_{1}}{2 \alpha_{0} h_{1}+\sinh 2 \alpha_{0} h_{1}} \int_{0}^{h_{l}} f(y) \cos \alpha_{0}\left(h_{1}-y\right) d y, \\
& -s_{n} A_{n}=\frac{4 \alpha_{n}}{2 \alpha_{n} h_{l}+\sin 2 \alpha_{n} h_{l}} \int_{0}^{h_{l}} f(y) \cos \alpha_{n}\left(h_{1}-y\right) d y, \\
& i \mu\left(1-R_{1}\right)=\frac{4 k_{0} \cosh k_{0} h_{2}}{2 k_{0} h_{2}+\sinh 2 k_{0} h_{2}} \int_{0}^{h_{l}} f(y) \cos k_{0}\left(h_{2}-y\right) d y, \\
& p_{n} B_{n}=\frac{4 k_{n}}{2 k_{n} h_{2}+\sin 2 k_{n} h_{2}} \int_{0}^{h_{l}} f(y) \cos k_{n}\left(h_{2}-y\right) d y .
\end{aligned}
$$

Using Eqs (3.6), (3.7), (3.9) in Eq.(3.5) and from Eq.(3.8), we find

$$
\begin{aligned}
& \int_{0}^{h_{l}} F_{l}(u) M_{l}(y, u) d u=\frac{\cosh k_{0}\left(h_{2}-y\right)}{\cosh k_{0} h_{2}}, \quad 0<y<h_{1}, \\
& \int_{0}^{h_{l}} F_{l}(y) \frac{\cosh k_{0}\left(h_{2}-y\right)}{\cosh k_{0} h_{2}} d y=C_{l}
\end{aligned}
$$

where

$$
F_{1}(u)=\frac{-f(u)}{1+R_{1}} \frac{4 k_{0} \cosh ^{2} k_{0} h_{2}}{\mu\left(2 k_{0} h_{2}+\sinh 2 k_{0} h_{2}\right)},
$$




$$
\begin{aligned}
& M_{l}(y, u)=\frac{\mu\left(2 k_{0} h_{2}+\sinh 2 k_{0} h_{2}\right)}{k_{0} \cosh ^{2} k_{0} h_{2}}\left[\frac{i \alpha_{0} \cosh \alpha_{0}\left(h_{1}-y\right) \cosh \alpha_{0}\left(h_{1}-u\right)}{\beta\left(2 \alpha_{0} h_{1}+\sinh 2 \alpha_{0} h_{1}\right)}+\right. \\
& \left.+\sum_{l}^{\infty}\left\{\frac{\alpha_{n} \cos \alpha_{n}\left(h_{1}-y\right) \cos \alpha_{n}\left(h_{1}-u\right)}{s_{n}\left(2 \alpha_{n} h_{1}+\sin 2 \alpha_{n} h_{1}\right)}+\frac{k_{n} \cos k_{n}\left(h_{2}-y\right) \cos k_{n}\left(h_{2}-u\right)}{p_{n}\left(2 k_{n} h_{2}+\sin 2 k_{n} h_{2}\right)}\right\}\right] \\
& C_{1}=\frac{-i\left(1-R_{1}\right)}{1+R_{1}}
\end{aligned}
$$

It may be noted that the function $F_{1}(y)$ and the constant $C_{1}$ are real. The integral Eq.(3.10) is to be solved by $(N+l)$ multi-term Galerkin approximations of $F_{l}(y)$ in terms of ultraspherical Gegenbauer polynomials $C_{2 n}^{1 / 6}\left(y / h_{l}\right)$ by noting the behavior of $F_{l}(y) \sim\left(h_{1}-y\right)^{-1 / 3}$ as $y \rightarrow h_{l}-0$ given by (cf. Kanoria et al. [19])

$$
F_{l}(y)=\sum_{n=0}^{N} a_{n} f_{n}(y), \quad 0<y<h_{l}
$$

where

$$
f_{n}(y)=-\frac{d}{d y} \exp (-K y) \int_{y}^{h_{l}} \exp (K t) \hat{f_{n}}(t) d t, 0<y<h_{l},
$$

with

$$
\hat{f}_{n}(y)=\frac{2^{7 / 6} \Gamma(1 / 6)(2 n) !}{\pi \Gamma(2 \mathrm{n}+1 / 3) h_{l}^{1 / 3}\left(h_{l}^{2}-y^{2}\right)^{1 / 3}} C_{2 n}^{1 / 6}\left(y / h_{l}\right)
$$

The unknown coefficients $a_{n}(n=0,1,2, \cdots, N)$ are obtained by solving the system of linear equations

$$
\sum_{n=0}^{N} a_{n} \Re_{n m}=d_{m}, \quad m=0,1,2, \cdots, N
$$

where

$$
\begin{aligned}
& \mathfrak{R}_{n m}=\frac{\mu\left(2 k_{0} h_{2}+\sinh 2 k_{0} h_{2}\right)}{k_{0} \cosh ^{2} k_{0} h_{2}}\left[4 ( - 1 ) ^ { n + m } \sum _ { r = 1 } ^ { \infty } \left\{\frac{k_{r} \cos ^{2} k_{r} h_{2}}{p_{r}\left(2 k_{r} h_{2}+\sin 2 k_{r} h_{2}\right)} \frac{J_{2 n+1 / 6}\left(k_{r} h_{2}\right) J_{2 m+1 / 6}\left(k_{r} h_{2}\right)}{\left(k_{r} h_{2}\right)^{1 / 3}}+\right.\right. \\
& \left.+\frac{\alpha_{r} \cos ^{2} \alpha_{r} h_{1}}{s_{r}\left(2 \alpha_{r} h_{1}+\sin 2 \alpha_{r} h_{1}\right)} \frac{J_{2 n+1 / 6}\left(\alpha_{r} h_{1}\right) J_{2 m+1 / 6}\left(\alpha_{r} h_{l}\right)}{\left(\alpha_{r} h_{1}\right)^{1 / 3}}\right\}+ \\
& \left.+\frac{i \alpha_{0} \cosh ^{2} \alpha_{0} h_{1}}{\beta\left(2 \alpha_{0} h_{1}+\sinh 2 \alpha_{0} h_{l}\right)} \frac{I_{2 n+1 / 6}\left(\alpha_{0} h_{1}\right) I_{2 m+1 / 6}\left(\alpha_{0} h_{l}\right)}{\left(\alpha_{0} h_{l}\right)^{1 / 3}}\right],
\end{aligned}
$$




$$
d_{m}=\frac{I_{2 m+1 / 6}\left(k_{0} h_{2}\right)}{\left(k_{0} h_{2}\right)^{1 / 6}}
$$

Once $a_{n}(n=0,1,2, \cdots, N)$ are solved, the real constant $C_{1}$ can be determined from Eq.(3.11)

$$
C_{l}=\sum_{n=0}^{N} a_{n} d_{n}
$$

Then $R_{l}$ can be found using Eq.(3.12) and $T_{1}$ can be found from Eqs (3.6) using (3.13) as

$$
\begin{aligned}
& R_{1}=\frac{1-i C_{1}}{1+i C_{1}}, \\
& T_{1}=\frac{i A \cosh \alpha_{0} h_{1}}{\left(\alpha_{0} h_{1}\right)^{1 / 6}} \sum_{n=0}^{N} a_{n} I_{2 n+1 / 6}\left(\alpha_{0} h_{l}\right)
\end{aligned}
$$

where

$$
A=\frac{\alpha_{0} \mu\left(1+R_{l}\right) \cosh \alpha_{0} h_{1}\left(2 k_{0} h_{2}+\sinh 2 k_{0} h_{2}\right)}{\beta k_{0} \cosh ^{2} k_{0} h_{2}\left(2 \alpha_{0} h_{1}+\sinh 2 \alpha_{0} h_{1}\right)} .
$$

If a simple harmonic progressive oblique wave train originating at $x \rightarrow+\infty$ is incident on the step, and is partially reflected and partially transmitted, the time harmonic progressive waves from positive infinity can be represented by the velocity potentials $\operatorname{Re}\left\{\varphi_{+}^{\text {inc }}(x, y) \exp \left(i \vartheta_{1} z-i \sigma t\right)\right\}$ where

$$
\varphi_{+}^{\mathrm{inc}}(x, y)=\frac{\cosh \alpha_{0}\left(h_{1}-y\right)}{\cosh \alpha_{0} h_{l}} \exp \left(-i \beta_{1} x\right),
$$

with $\vartheta_{l}=\alpha_{0} \sin \alpha, \beta_{l}=\alpha_{0} \cos \alpha$.

If the resulting motion is described by the velocity potential $\operatorname{Re}\left\{\varphi(x, y) \exp \left(i \vartheta_{1} z-i \sigma t\right)\right\}$, then $\varphi$ satisfies

$$
\nabla^{2} \varphi-\vartheta_{l}^{2} \varphi=0 \text { in the fluid region, }
$$

and the conditions from Eqs (2.4) to (2.7).

The behavior of $\varphi(x, y)$ at infinity gives

$$
\varphi(x, y) \rightarrow\left\{\begin{array}{l}
T_{2} \frac{\cosh k_{0}\left(h_{2}-y\right)}{\cosh k_{0} h_{2}} \exp \left(-i \mu_{1} x\right) \text { as } x \rightarrow-\infty \\
\frac{\cosh \alpha_{0}\left(h_{1}-y\right)}{\cosh \alpha_{0} h_{1}}\left\{\exp \left(-i \beta_{1} x\right)+R_{2} \exp \left(i \beta_{1} x\right)\right\} \text { as } x \rightarrow+\infty
\end{array}\right.
$$


where $\mu_{1}^{2}=k_{0}^{2}-\vartheta_{1}^{2}$ and $T_{2}, R_{2}$ are the unknown transmission and reflection coefficients to be determined. A solution for $\varphi(x, y)$ satisfying Eqs (3.19), (2.4), (2.5) and (3.20) can be represented as

$$
\varphi(x, y) \rightarrow\left\{\begin{array}{l}
T_{2} \frac{\cosh k_{0}\left(h_{2}-y\right)}{\cosh k_{0} h_{2}} \exp \left(-i \mu_{1} x\right)+\sum_{l}^{\infty} D_{n} \cos k_{n}\left(h_{2}-y\right) \exp \left(p_{n}^{\prime} x\right), \quad x \leq 0, \\
\frac{\cosh \alpha_{0}\left(h_{1}-y\right)}{\cosh \alpha_{0} h_{1}}\left\{\exp \left(-i \beta_{1} x\right)+R_{2} \exp \left(i \beta_{1} x\right)\right\}+\sum_{l}^{\infty} C_{n} \cos \alpha_{n}\left(h_{1}-y\right) \exp \left(-s_{n}^{\prime} x\right), \quad x \geq 0
\end{array}\right.
$$

where $s_{n}^{\prime}=\sqrt{\alpha_{n}^{2}+\vartheta_{l}^{2}}, p_{n}^{\prime}=\sqrt{k_{n}^{2}+\vartheta_{l}^{2}}$.

Using Eq.(3.21) in Eqs (3.1) and (3.2), we find

$$
\begin{aligned}
& f(y)=-i \mu_{1} T_{2} \frac{\cosh k_{0}\left(h_{2}-y\right)}{\cosh k_{0} h_{2}}+\sum_{1}^{\infty} p_{n}^{\prime} D_{n} \cos k_{n}\left(h_{2}-y\right), 0<y<h_{2}, \\
& =i \beta_{1}\left(R_{2}-1\right) \frac{\cosh \alpha_{0}\left(h_{1}-y\right)}{\cosh \alpha_{0} h_{1}}-\sum_{1}^{\infty} s_{n}^{\prime} C_{n} \cos \alpha_{n}\left(h_{1}-y\right), \quad 0<y<h_{1}
\end{aligned}
$$

and

$$
\begin{aligned}
& T_{2} \frac{\cosh k_{0}\left(h_{2}-y\right)}{\cosh k_{0} h_{21}}+\sum_{1}^{\infty} D_{n} \cos k_{n}\left(h_{2}-y\right)=\left(1+R_{2}\right) \frac{\cosh \alpha_{0}\left(h_{1}-y\right)}{\cosh \alpha_{0} h_{12}}+ \\
& +\sum_{l}^{\infty} C_{n} \cos \alpha_{n}\left(h_{1}-y\right), \quad 0<y<h_{1} .
\end{aligned}
$$

Use of Havelock's [18] inversion theorem in Eq.(3.22) produces

$$
\begin{aligned}
& -i \mu_{1} T_{2}=\frac{4 k_{0} \cosh k_{0} h_{2}}{2 k_{0} h_{2}+\sinh 2 k_{0} h_{2}} \int_{0}^{h_{l}} f(y) \cos k_{0}\left(h_{2}-y\right) d y, \\
& -s_{n}^{\prime} C_{n}=\frac{4 \alpha_{n}}{2 \alpha_{n} h_{l}+\sin 2 \alpha_{n} h_{l}} \int_{0}^{h_{l}} f(y) \cos \alpha_{n}\left(h_{l}-y\right) d y, \\
& i \beta_{1}\left(R_{2}-1\right)=\frac{4 \alpha_{0} \cosh \alpha_{0} h_{1}}{2 \alpha_{0} h_{12}} \int_{0}^{h_{1}} f(y) \sinh 2 \alpha_{0} h_{12} \cos \alpha_{0}\left(h_{1}-y\right) d y, \\
& p_{n}^{\prime} D_{n}=\frac{4 k_{n}}{2 k_{n} h_{2}+\sin 2 k_{n} h_{2}} \int_{0}^{h_{1}} f(y) \cos k_{n}\left(h_{2}-y\right) d y .
\end{aligned}
$$


Using Eqs (3.24), (3.25), (3.27) in Eq.(3.23) and from Eq.(3.26), we find

$$
\begin{aligned}
& \int_{0}^{h_{1}} F_{2}(u) M_{2}(y, u) d u=\frac{\cosh \alpha_{0}\left(h_{1}-y\right)}{\cosh \alpha_{0} h_{1}}, \quad 0<y<h_{1}, \\
& \int_{0}^{h_{l}} F_{2}(y) \frac{\cosh \alpha_{0}\left(h_{1}-y\right)}{\cosh \alpha_{0} h_{1}} d y=C_{2}
\end{aligned}
$$

where

$$
\begin{aligned}
& F_{2}(u)=\frac{f(u)}{1+R_{2}} \frac{4 \alpha_{0} \cosh ^{2} \alpha_{0} h_{1}}{\beta_{1}\left(2 \alpha_{0} h_{1}+\sinh 2 \alpha_{0} h_{1}\right)}, \\
& M_{2}(y, u)=\frac{\beta_{1}\left(2 \alpha_{0} h_{1}+\sinh 2 \alpha_{0} h_{1}\right)}{\alpha_{0} \cosh ^{2} \alpha_{0} h_{1}}\left[\frac{i k_{0} \cosh k_{0}\left(h_{2}-y\right) \cosh k_{0}\left(h_{2}-u\right)}{\mu_{1}\left(2 k_{0} h_{2}+\sinh 2 k_{0} h_{2}\right)}+\right. \\
& +\sum_{1}^{\infty}\left\{\frac{\alpha_{n} \cos \alpha_{n}\left(h_{1}-y\right) \cos \alpha_{n}\left(h_{1}-u\right)}{s_{n}^{\prime}\left(2 \alpha_{n} h_{1}+\sin 2 \alpha_{n} h_{1}\right)}+\frac{k_{n} \cos k_{n}\left(h_{2}-y\right) \cos k_{n}\left(h_{2}-u\right)}{p_{n}^{\prime}\left(2 k_{n} h_{2}+\sin 2 k_{n} h_{2}\right)}\right\} . \\
& C_{2}=\frac{i\left(R_{2}-1\right)}{R_{2}+1} .
\end{aligned}
$$

It may be noted that the function $F_{2}(y)$ and the constant $C_{2}$ are real. The integral Eq.(3.28) is to be solved by $(N+l)$ multi-term Galerkin approximations of $F_{2}(y)$ in terms of ultraspherical Gegenbauer polynomials $C_{2 n}^{1 / 6}\left(y / h_{1}\right)$ by noting the behavior of $F_{2}(y) \sim\left(h_{1}-y\right)^{-1 / 3}$ as $y \rightarrow h_{1}-0$ given by (cf. Kanoria et al. [19])

$$
F_{2}(y)=\sum_{n=0}^{N} a_{n}^{\prime} f_{n}(y), \quad 0<y<h_{1}
$$

where

$$
f_{n}(y)=-\frac{d}{d y} \exp (-K y) \int_{y}^{h_{l}} \exp (K t) \hat{f_{n}}(t) d t, 0<y<h_{l}
$$

with

$$
\hat{f}_{n}(y)=\frac{2^{7 / 6} \Gamma(1 / 6)(2 n) !}{\pi \Gamma(2 \mathrm{n}+1 / 3) h_{l}^{1 / 3}\left(h_{l}^{2}-y^{2}\right)^{1 / 3}} C_{2 n}^{1 / 6}\left(y / h_{l}\right)
$$

The unknown coefficients $a_{n}^{\prime}(n=0,1,2, \cdots, N)$ are obtained by solving the system of linear equations 


$$
\sum_{n=0}^{N} a_{n}^{\prime} \Re_{n m}^{\prime}=d_{m}^{\prime}, \quad m=0,1,2, \cdots, N
$$

where

$$
\begin{gathered}
\mathfrak{R}_{n m}^{\prime}=\frac{\beta_{1}\left(2 \alpha_{0} h_{1}+\sinh 2 \alpha_{0} h_{1}\right)}{\alpha_{0} \cosh ^{2} \alpha_{0} h_{12}}\left[4 ( - 1 ) ^ { n + m } \sum _ { r = 1 } ^ { \infty } \left\{\frac{\alpha_{r} \cos ^{2} \alpha_{r} h_{12}}{s_{r}^{\prime}\left(2 \alpha_{r} h_{12}+\sin 2 \alpha_{r} h_{12}\right)} \frac{J_{2 n+1 / 6}\left(\alpha_{r} h_{1}\right) J_{2 m+1 / 6}\left(\alpha_{r} h_{l}\right)}{\left(\alpha_{r} h_{1}\right)^{1 / 3}}+\right.\right. \\
\left.\left.+\frac{k_{r} \cos ^{2} k_{r} h_{2}}{p_{r}^{\prime}\left(2 k_{r} h_{2}+\sin 2 k_{r} h_{2}\right)} \frac{J_{2 n+1 / 6}\left(k_{r} h_{2}\right) J_{2 m+1 / 6}\left(k_{r} h_{2}\right)}{\left(k_{r} h_{2}\right)^{1 / 3}}\right\}+\frac{i k_{0} \cosh ^{2} k_{0} h_{2}}{\mu_{l}\left(2 k_{0} h_{2}+\sinh 2 k_{0} h_{2}\right)} \frac{I_{2 n+1 / 6}\left(k_{0} h_{2}\right) I_{2 m+1 / 6}\left(k_{0} h_{2}\right)}{\left(k_{0} h_{2}\right)^{1 / 3}}\right], \\
d_{m}^{\prime}=\frac{I_{2 m+1 / 6}\left(\alpha_{0} h_{1}\right)}{\left(\alpha_{0} h_{1}\right)^{1 / 6}} .
\end{gathered}
$$

Once $a_{n}^{\prime}(n=0,1,2, \cdots, N)$ are solved, the real constant $C_{2}$ can be determined from Eq.(3.29)

$$
C_{2}=\sum_{n=0}^{N} a_{n}^{\prime} d_{n}^{\prime}
$$

Then $R_{2}$ can be found using Eq.(3.30) and $T_{2}$ can be found from Eq.(3.24) using (3.31) as

$$
\begin{aligned}
& R_{2}=\frac{1-i C_{2}}{1+i C_{2}}, \\
& T_{2}=\frac{i B \cosh k_{0} h_{2}}{\left(k_{0} h_{2}\right)^{1 / 6}} \sum_{n=0}^{N} a_{n}^{\prime} I_{2 n+1 / 6}\left(k_{0} h_{2}\right)
\end{aligned}
$$

where

$$
B=\frac{k_{0} \beta_{1}\left(1+R_{2}\right) \cosh k_{0} h_{2}\left(2 \alpha_{0} h_{1}+\sinh 2 \alpha_{0} h_{1}\right)}{\mu_{1} \alpha_{0} \cosh ^{2} \alpha_{0} h_{1}\left(2 k_{0} h_{2}+\sinh 2 k_{0} h_{2}\right)} .
$$

\section{Numerical results}

Multi-term Galerkin approximations are used to obtain a numerical estimate for $\left|R_{l}\right|,\left|T_{1}\right|$ and $\left|R_{2}\right|,\left|T_{2}\right|$. In the numerical computations we take at most six terms to produce a fairly accurate numerical estimate for $\left|R_{1}\right|,\left|T_{1}\right|$ and $\left|R_{2}\right|,\left|T_{2}\right|$.

We display a representative set of numerical estimates for $\left|R_{1}\right|,\left|T_{1}\right|$ and $\left|R_{2}\right|,\left|T_{2}\right|$ in Tab.1 and Tab.2, taking $N=0,1,2,3,4$ and 5 in the $(N+1)$ - term of Galerkin approximations and some particular values of the different parameters and the wave numbers. 
Table 1

\begin{tabular}{|c|c|c|c|c|}
\hline & \multicolumn{4}{|c|}{$h_{1} / h_{2}=0.5, K h_{2}=0.2, \alpha=0^{0}$} \\
\hline & $\left|R_{1}\right|$ & $\left|R_{2}\right|$ & $\left|T_{1}\right|$ & $\left|T_{2}\right|$ \\
\hline 0 & 0.236756 & 0.236756 & 1.126449 & 0.837985 \\
\hline 1 & 0.234018 & 0.234018 & 1.127218 & 0.838556 \\
\hline 2 & 0.233981 & 0.233981 & 1.127228 & 0.838564 \\
\hline 3 & 0.233979 & 0.233979 & 1.127229 & 0.838565 \\
\hline 4 & 0.233979 & 0.233979 & 1.127229 & 0.838564 \\
\hline 5 & 0.233979 & 0.233979 & 1.127229 & 0.838564 \\
\hline \multicolumn{5}{|c|}{$h_{1} / h_{2}=0.5, K h_{2}=0.2, \alpha=30^{0}$} \\
\hline & $\left|R_{1}\right|$ & $\left|R_{2}\right|$ & $\left|T_{1}\right|$ & $\left|T_{2}\right|$ \\
\hline 0 & 0.201124 & 0.157099 & 1.094152 & 0.934879 \\
\hline 1 & 0.198444 & 0.154480 & 1.094762 & 0.935270 \\
\hline 2 & 0.198408 & 0.154444 & 1.094771 & 0.935275 \\
\hline 3 & 0.198406 & 0.154442 & 1.094771 & 0.935275 \\
\hline 4 & 0.198405 & 0.154442 & 1.094771 & 0.935275 \\
\hline 5 & 0.198405 & 0.154442 & 1.094771 & 0.935275 \\
\hline
\end{tabular}

Table 2

\begin{tabular}{|c|c|c|c|c|}
\hline & \multicolumn{4}{|c|}{$h_{1} / h_{2}=0.5, K h_{2}=1.8, \alpha=0^{0}$} \\
\hline & $\left|R_{l}\right|$ & $\left|R_{2}\right|$ & $\left|T_{1}\right|$ & $\left|T_{2}\right|$ \\
\hline 0 & 0.259046 & 0.259046 & 0.934748 & 0.998018 \\
\hline 1 & 0.259317 & 0.259317 & 0.934678 & 0.997943 \\
\hline 2 & 0.259138 & 0.259138 & 0.934724 & 0.997992 \\
\hline 3 & 0.259127 & 0.259127 & 0.934727 & 0.997995 \\
\hline 4 & 0.259126 & 0.259126 & 0.934727 & 0.997996 \\
\hline 5 & 0.259126 & 0.259126 & 0.934727 & 0.997996 \\
\hline \multicolumn{5}{|c|}{$h_{1} / h_{2}=0.5, K h_{2}=1.8, \alpha=30^{0}$} \\
\hline & $\left|R_{l}\right|$ & $\left|R_{2}\right|$ & $\left|T_{1}\right|$ & $\left|T_{2}\right|$ \\
\hline 0 & 0.217419 & 0.201057 & 0.923292 & 1.049337 \\
\hline 1 & 0.218222 & 0.201998 & 0.923122 & 1.049129 \\
\hline 2 & 0.218054 & 0.201835 & 0.923159 & 1.049165 \\
\hline 3 & 0.218040 & 0.201825 & 0.923161 & 1.049167 \\
\hline 4 & 0.218039 & 0.201824 & 0.923161 & 1.049167 \\
\hline 5 & 0.218039 & 0.201824 & 0.923161 & 1.049167 \\
\hline
\end{tabular}

It is observed from Tabs 1 and 2 that the computed results for $\left|R_{1}\right|,\left|T_{1}\right|$ and $\left|R_{2}\right|,\left|T_{2}\right|$ converge very rapidly with $N$, and for $N \geq 3$ an accuracy of almost six decimal places is observed. It appears that the present numerical procedure for the numerical computations of reflection and transmission coefficients is quite efficient. We also note from these tables that for normal incidence of the wave train $\left(\alpha=0^{0}\right)$, $\left|R_{1}\right|=\left|R_{2}\right|\left(=|R|\right.$,say) and $\left|T_{1} T_{2}\right|=1-|R|^{2}$. Similar observations were made by Newman (1965) for the infinite step problem. 


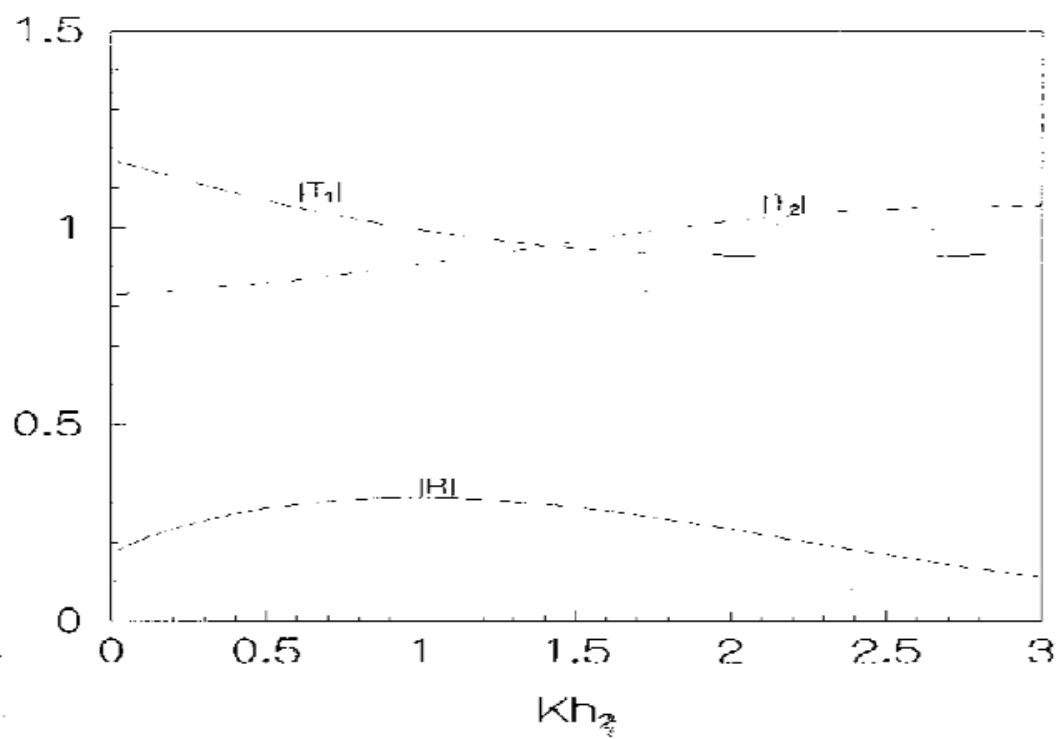

Fig.2. Reflection and transmission coefficients for $h_{1} / h_{2}=0.5, \alpha=0^{0}$.

The computed results for $|R|$ and $\left|T_{1}\right|,\left|T_{2}\right|$ are plotted in Fig. 2 against $K h_{2}$ for $h_{1} / h_{2}=0.5, \alpha=0^{0}$. It is observed that $|R|$ increases for some moderate values of $K h_{2}$ and then decreases as $K h_{2}$ increases. On the other hand, $\left|T_{1}\right|$ decreases and $\left|T_{2}\right|$ increases as $K h_{2}$ increases and $\left|T_{1}\right|=\left|T_{2}\right| \approx 1.0$ for some particular value of $K h_{2} \approx 1.5$. Also, it is observed that $|R| \rightarrow 0$ and $\left|T_{1}\right| \rightarrow 1,\left|T_{2}\right| \rightarrow 1$ as $K h_{2} \rightarrow \infty$. These type of observations are quite plausible because for small $K h_{2}$, waves penetrate on the step and for large $K h_{2}$, waves are mainly confined at the free surface, so that waves are totally transmitted through the gap.

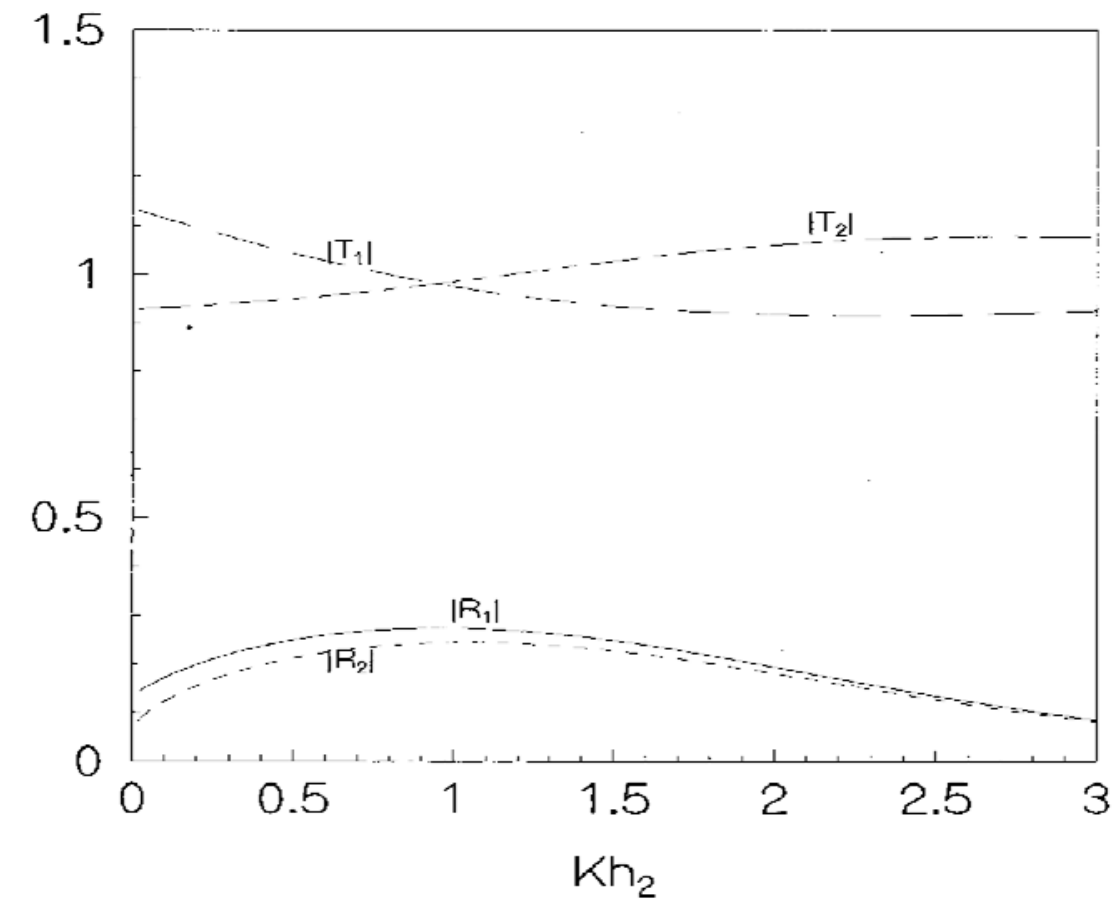

Fig.3. Reflection and transmission coefficients for $h_{1} / h_{2}=0.5, \alpha=30^{0}$. 
In Fig.3, the computed results for $\left|R_{1}\right|,\left|R_{2}\right|$ and $\left|T_{1}\right|,\left|T_{2}\right|$ are plotted against $K h_{2}$ for $h_{1} / h_{2}=0.5, \alpha=30^{\circ}$. It is observed that $\left|R_{1}\right|>\left|R_{2}\right|$ for all $K h_{2}$. It is quite obvious because the reflection coefficient for penetration from the front side of the step will be greater than penetration from the back side of the step. $\left|R_{1}\right|$ and $\left|R_{2}\right|$ first increases then decreases as $K h_{2}$ increases and tending to zero as $K h_{2} \rightarrow \infty$. Also, $\left|T_{1}\right|$ decreases and $\left|T_{2}\right|$ increases as $K h_{2}$ increases and $\left|T_{1}\right|=\left|T_{2}\right| \approx 1.0$ for some particular value of $K h_{2} \approx 1.0$. It is also observed that $\left|T_{1}\right| \rightarrow 1,\left|T_{2}\right| \rightarrow 1$ as $K h_{2} \rightarrow \infty$.

\section{Conclusion}

The method of multi-term Galerkin approximations in terms of ultra spherical Gegenbauer polynomials has been utilized here to obtain very accurate numerical estimates for the reflection and transmission coefficients in the water wave scattering problem of an obliquely incident surface wave train on an obstacle in the form of a finite step. By choosing only five terms in the Galerkin approximations, we achieve accuracy almost to the sixth digit after the deumol point. In the numerical estimates for the reflection and transmission coefficients. The numerical results are illustrated in tables and figures. For normal incidence of the surface wave train, the results are compared with the known results available in the literature for similar problems and very good agreement is achieved.

\section{Acknowledgement} Project.

This work is supported by the University Grants Commission, New Delhi, through a Minor Research

\section{Nomenclature}

$$
\begin{aligned}
g & - \text { gravity } \\
h_{1} & - \text { depth of the upper step } \\
h_{2} & \text { - depth of the lower step } \\
K & \text { - wave number } \\
R_{1}, R_{2} & \text { - reflection coefficients } \\
T_{1}, T_{2} & \text { - transmission coefficient } \\
t & \text { - time } \\
x & \text { - horizontal distance } \\
y & \text { - vertical distance } \\
\sigma & \text { - wave frequency } \\
\varphi & - \text { velocity potential }
\end{aligned}
$$

\section{References}

[1] Dean W.R. (1945): On the reflection of surface waves by a submerged plane barrier. - Proc. Camb. Phil. Soc., vol.41, pp.231-238.

[2] Ursell F. (1947): The effect of a fixed barrier on surface waves in deep water. - Proc. Camb. Phil. Soc., vol.43, pp.374-382.

[3] Evans D.V. (1970): Diffraction of water waves by a submerged vertical plate. - J. Fluid Mech., vol.40, pp.433-451.

[4] Porter D. (1972): The transmission of surface waves through a gap in a vertical barrier. - Proc. Camb. Phil. Soc., vol.71, pp.411-422. 
[5] Mandal B.N. and Dolai D.P. (1994): Oblique water wave diffraction by thin vertical barriers in water of uniform finite depth. - Appl. Ocean Res., vol.16, pp.195-203.

[6] Roseau M. (1976): Asymptotic wave theory. - North Holland, pp.311-347.

[7] Kreisel G. (1949): Surface waves. - Quart. Appl. Math., vol.7, pp.21-44.

[8] Fitz-Gerald G.F. (1976): The reflection of plane gravity waves traveling in water of variable depth. - Phil. Trans. Roy. Soc. Lond., vol.34, pp.49-89.

[9] Hamilton J. (1977): Differential equations for long period gravity waves on fluid of rapidly varying depth. - J. Fluid Mech., vol.83, pp.289-310.

[10] Newman J.N. (1965): Propagation of water waves over an infinite step. - J. Fluid Mech., vol.23, pp.399-415.

[11] Miles J.W. (1967): Surface wave scattering matrix for a shelf. - J. Fluid Mech., vol.28, pp.755-767.

[12] Mandal B.N. and Gayen, Rupanwita (2006): Water wave scattering by bottom undulations in the presence of a thin partially immersed barrier. - Appl. Ocean Res., vol.28, pp.113-119.

[13] Dolai D.P. and Dolai P. (2010): Interface wave diffraction by bottom undulations in the presence of a thin plate submerged in lower fluid. - Int. J. Appl. Mech. and Engg. vol.15, pp.1017-1036.

[14] Stoker J.J. (1957): Water Waves. - New York: Interscience.

[15] Wehausen J.V. and Laiton E.V. (1960): Surface Waves. - Handbuch der Physik: Springer.

[16] Bartholomeusz E.F. (1958): The reflection of long waves at a step. - Proc. Camb. Phil. Soc., vol.54, pp.106-118.

[17] Evans D.V. and McIver P. (1984): Edge waves over a shelf: full linear theory. - J. Fluid Mech., vol.142, pp.7995.

[18] Havelock T.H. (1929): Forced surface waves on water. - Phil. Mag., vol.8, pp.569-576.

[19] Kanoria M., Dolai D.P. and Mandal B.N. (1999): Water wave scattering by thick vertical barriers. - J. Eng. Math., vol.35, pp.361-384.

Received: April 12, 2016

Revised: October 15, 2016 